\title{
Characterization of Soil Particle Size Distribution with a Fractal Model in the Desertified Regions of Northern China
}

\author{
Guang-Lei GAO ${ }^{1,2}$, Guo-Dong DING ${ }^{1,2}$, Yuan-Yuan $\mathrm{ZHAO}^{1,2}$, Bin WU ${ }^{1,2}$, \\ Yu-Qing ZHANG ${ }^{1,2}$, Jian-Bin GUO ${ }^{1,2}$, Shu-Gao QIN ${ }^{1,2}$, Yan-Feng BAO ${ }^{1}$, \\ Ming-Han $\mathrm{YU}^{1}$, and Yun-Dong $\mathrm{LIU}^{1}$ \\ ${ }^{1}$ Yanchi Research Station, School of Soil and Water Conservation, \\ Beijing Forestry University, Beijing, P.R. China \\ ${ }^{2}$ Key Laboratory of Soil and Water Conservation and Desertification Combating, \\ Ministry of Education, Beijing Forestry University, Beijing, P.R. China; \\ e-mail: dingguodong@bjfu.edu.cn
}

\begin{abstract}
We constructed an aeolian soil database across arid, semi-arid, and dry sub-humid regions, China. Soil particle size distribution was measured with a laser diffraction technique, and fractal dimensions were calculated. The results showed that: (i) the predominant soil particle size distributed in fine and medium sand classifications, and fractal dimensions covered a wide range from 2.0810 to 2.6351 ; (ii) through logarithmic transformations, fractal dimensions were significantly positive correlated with clay and silt contents $\left(R^{2}=0.81\right.$ and $\left.0.59, P<0.01\right)$, and significantly negative correlated with sand content $\left(R^{2}=0.50, P<0.01\right)$; (3) hierarchical cluster analysis divided the plots into three types which were similar to sand dune types indicating desertification degree. In a large spatial scale, fractal dimensions are still sensitive to wind-induced desertification. Therefore, we highly recommend that fractal dimension be used as a reliable and quantitative parameter to monitor soil environment changes in desertified regions. This improved information provides a firm basis for better understanding of desertification processes.
\end{abstract}

Key words: desertification, aeolian soil, particle size distribution, fractal dimension. 


\section{INTRODUCTION}

Desertification means land degradation in arid, semi-arid, and dry sub-humid areas resulting from various factors including climatic variations and human activities (UNEP 1994). In China, desertified lands usually lie in the regions above $35^{\circ} \mathrm{N}$ with an annual precipitation $<450 \mathrm{~mm}$ (Wang et al. 2008). The latest Bulletin by the State Forestry Administration, P.R. China, has declared that desertification regions in China cover 2.62 million $\mathrm{km}^{2}$, accounting for more than $27 \%$ of the total landmass (SFA 2011). Desert expansions and remediation strategies have already been studied in China (Chen and Tang 2005, Wang et al. 2008). However, land desertification does not only cause expansions of desert landscape, but also soil structure damage and functional losses on a smaller scale (Zha and Gao 1997). Therefore, the recovery of soil structures and functions to sustain plant and animal life and desert ecosystem development has drawn increasing attention recently (Duan et al. 2004, Li et al. 2007, Chen and Duan 2009, Jiao et al. 2011).

Soil particle size distribution (PSD) is one of the fundamental attributes of soil systems (Hillel 1980). Soil PSD affects the movement and retention of water, solutes, heat, and air ( $\mathrm{Su}$ et al. 2004), and is widely used as a basis for estimating soil texture, organic carbon, nutrients, and hydraulic properties (Tyler and Wheatcraft 1990, Skaggs et al. 2001 Arrouays et al. 2006, Reynolds et al. 2006). Therefore, characterizing soil PSD with a single index that retains more information, as well as changes that occur due to desertification, is an approach of great interest when dealing with soil data (Su et al. 2004).

Numerous studies have developed mathematical models for estimating soil PSD (Buchan 1989, Nemes et al. 1999, Skaggs et al. 2001, Hwang et al. 2002). However, in these studies, the irregular and nonlinear structures of soil systems have been simply ignored as indescribable, although they could provide a much better representation of many natural phenomena (Mandelbrot 1983). In the 1960s, Mandelbrot (1967) created fractal geometry to describe the complex natural world, characterized by irregularity, selfsimilarity, and nonlinearity, and firstly provided a fractal model to describe soil PSD and its variations (Mandelbrot 1983). Following this, fractal arithmetic was more and more often applied to identify soil PSD and additional environmental changes (Turcotte 1986, Tyler and Wheatcraft 1992, Crawford et al. 1993, Wang et al. 2005, Gao et al. 2014a). Among these studies, the common feature of fractal measurements is the use of fractal dimensions to characterize soil PSD (Gui et al. 2010). The latest studies of soil systems on the fractal dimensions of PSD have demonstrated that fractal dimensions significantly correlate with the various soil particles contents, and imply the variations in soil porosity, organic matter, and nutrient content following a 
linear trend (Su et al. 2004, Liu et al. 2009, Gui et al. 2010, Jin et al. 2013, Gao et al. 2014b). Fractal dimensions are considered to be more sensitive than soil texture, organic matter, as well as fertility in reflecting the variations in soil systems (Su et al. 2004, Fu et al. 2009), and become important in understanding and quantifying soil degradation and dynamics on different spatial scales (Jin et al. 2013). However, former studies have always focused on the variations in fractal dimensions on a smaller spatial scale; the uncertainty of variation on a larger spatial scale creates space for further developments of the use of fractal dimensions.

In desertification regions, wind-induced changes in soil PSD are drastic. Frequent and intense wind erosion sorts soil fractions, removing fine particles and leaving coarse-textured soil behind (Lobe et al. 2001). In this paper, we analyzed soil PSD and the fractal dimensions of topsoil from the desertified regions of northern China. The objectives of this study were: (i) to characterize soil PSD using fractal dimensions, (ii) to examine if fractal dimensions of soil PSD can represent the variations in soil systems over a large spatial scale, and (iii) additionally to assess desertification trends and the efforts of anti-desertification solutions in desertified regions of northern China.

\section{MATERIAL AND METHODS}

\subsection{Soil information}

A soil database was established from the field-based investigation of aeolian topsoil. It included 39 sampling plots from 7 field stations covering Horqin Sandland, Mu Us Sandland, Kubuqi Desert, Tengger Desert, Hexi Desert, and Gurbantunggut Desert across arid, semi-arid, and dry sub-humid regions of northern China (Table 1). In each sampling field, 3 sub-plots were selected for topsoil sample collection; in each sub-plot, 5 topsoil samples were randomly collected at a depth of $0-5 \mathrm{~cm}$. Additionally, the sampling positions were all on the flat tops of sand dunes to eliminate the effects of microphysiognomy. In total, 585 topsoil samples qualified.

\subsection{Soil analysis}

All soil samples were air-dried and passed through a $2 \mathrm{~mm}$ sieve prior to laboratory analysis. To indentify soil PSD information, the topsoil samples were pretreated in an $\mathrm{H}_{2} \mathrm{O}_{2}$ solution $(30 \%$, w/w) to destroy organic matter. Then, soil aggregates were dispersed by adding sodium hexametaphosphate (NaHMP) and sonicatied for $30 \mathrm{~s}$ (Wang et al. 2006, Gui et al. 2010). Finally, soil PSD data was obtained with a laser diffraction technique using a Malvern Instrument MS 2000 (Malvern, England) with a measurement range and error of $0.02-2000 \mu \mathrm{m}$ and $<2 \%$, respectively. Each sample was meas- 
Table 1

General information of the selected plots

in arid and semi-arid regions of northern China

\begin{tabular}{|c|c|c|c|c|c|c|c|c|}
\hline Plot & Land use & $\begin{array}{l}\text { Sand } \\
\text { dune }\end{array}$ & Vegetation type & \begin{tabular}{|c|} 
Planta- \\
tion \\
duration \\
[years]
\end{tabular} & Soil type & $\begin{array}{c}\text { Altitude } \\
{[\mathrm{m}]}\end{array}$ & Location & Region \\
\hline 1 & Woodland & Fixed & Pinus sylvestnis & 35 & $\begin{array}{c}\text { Aeolian } \\
\text { soil }\end{array}$ & 207 & $\begin{array}{l}\text { N42 } 41.15^{\prime}, \\
\text { E122 } \\
\end{array}$ & \begin{tabular}{|c|} 
Horqin \\
Sandland
\end{tabular} \\
\hline 2 & Woodland & Fixed & Pinus densiflora & 35 & $\begin{array}{l}\text { Aeolian } \\
\text { soil }\end{array}$ & 209 & $\begin{array}{l}\text { N42 } 41.03^{\prime}, \\
\text { E122 }\end{array}$ & $\begin{array}{c}\text { Horqin } \\
\text { Sandland }\end{array}$ \\
\hline 3 & Woodland & Fixed & $\begin{array}{l}\text { Pinus densiflora } \\
\text { var zhanguensis }\end{array}$ & 22 & $\begin{array}{l}\text { Aeolian } \\
\text { soil }\end{array}$ & 203 & $\begin{array}{l}\text { N42 }{ }^{\circ} 41.22^{\prime}, \\
\text { E122 }\end{array}$ & \begin{tabular}{|c|} 
Horqin \\
Sandland
\end{tabular} \\
\hline 4 & Grassland & $\begin{array}{c}\text { Semi- } \\
\text { mobile }\end{array}$ & $\begin{array}{l}\text { Caragana } \\
\text { microphylla }\end{array}$ & - & $\begin{array}{c}\text { Aeolian } \\
\text { soil }\end{array}$ & 244 & $\begin{array}{l}\text { N42 }{ }^{\circ} 48.10, \\
\text { E122 } \\
\end{array}$ & \begin{tabular}{|c|} 
Horqin \\
Sandland
\end{tabular} \\
\hline 5 & Farmland & - & Zea mays & 25 & $\begin{array}{c}\text { Aeolian } \\
\text { soil } \\
\end{array}$ & 240 & $\begin{array}{l}\text { N42 } 48.07^{\prime}, \\
\text { E122 } \\
\end{array}$ & $\begin{array}{c}\text { Horqin } \\
\text { Sandland }\end{array}$ \\
\hline 6 & Grassland & $\begin{array}{c}\text { Semi- } \\
\text { mobile }\end{array}$ & $\begin{array}{l}\text { Arten } \\
\text { haloo }\end{array}$ & - & $\begin{array}{l}\text { Aeolian } \\
\text { soil }\end{array}$ & 358 & $\begin{array}{l}\mathrm{N} 42^{\circ} 56.40^{\prime}, \\
\mathrm{E} 120^{\circ} 42.97^{\prime}\end{array}$ & \begin{tabular}{|c|} 
Horqin \\
Sandland
\end{tabular} \\
\hline 7 & Woodland & \begin{tabular}{|c|} 
Semi- \\
mobile
\end{tabular} & Populus simonii & 14 & $\begin{array}{c}\text { Aeolian } \\
\text { soil } \\
\end{array}$ & 435 & $\begin{array}{l}\text { N42 } 33.18^{\prime}, \\
\text { E120 } 27.23^{\prime} \\
\end{array}$ & $\begin{array}{c}\text { Horqin } \\
\text { Sandland } \\
\end{array}$ \\
\hline 8 & Woodland & $\begin{array}{c}\text { Semi- } \\
\text { mobile }\end{array}$ & $\begin{array}{l}\text { P. simonii, } \\
\text { Hedysarum } \\
\text { fruticosum }\end{array}$ & 7 & $\begin{array}{l}\text { Aeolian } \\
\text { soil }\end{array}$ & 625 & $\begin{array}{l}\text { N42 } 23.83^{\prime}, \\
\text { E119 }\end{array}$ & $\begin{array}{c}\text { Horqin } \\
\text { Sandland }\end{array}$ \\
\hline 9 & Shrubland & Fixed & H. frut & 11 & $\begin{array}{c}\text { Aeolian } \\
\text { soil }\end{array}$ & 611 & $\begin{array}{l}\text { N42 } 23.89^{\prime}, \\
\text { E119 } \\
\end{array}$ & \begin{tabular}{|c|} 
Horqin \\
Sandland
\end{tabular} \\
\hline 10 & Shrubland & $\begin{array}{c}\text { Semi- } \\
\text { mobile }\end{array}$ & C. microphylla & 11 & $\begin{array}{c}\text { Aeolian } \\
\text { soil }\end{array}$ & 610 & $\begin{array}{l}\text { N42 } 23.89^{\prime}, \\
\text { E119 } \\
\end{array}$ & \begin{tabular}{|c|} 
Horqin \\
Sandland
\end{tabular} \\
\hline 11 & Shrubland & $\begin{array}{c}\text { Semi- } \\
\text { mobile }\end{array}$ & C. microphylla & 29 & $\begin{array}{c}\text { Aeolian } \\
\text { soil }\end{array}$ & 491 & $\begin{array}{l}\text { N42 }{ }^{\circ} 59.94^{\prime}, \\
\text { E119 } \\
\end{array}$ & $\begin{array}{c}\text { Horqin } \\
\text { Sandland }\end{array}$ \\
\hline 12 & - & Mobile & \begin{tabular}{|l|} 
Salix gordejevii, \\
H. fruticosum \\
\end{tabular} & 16 & $\begin{array}{c}\text { Aeolian } \\
\text { soil }\end{array}$ & 581 & $\begin{array}{l}\mathrm{N}^{\prime} 43^{\circ} 04.27^{\prime}, \\
\mathrm{E} 119^{\circ} 16.81^{\prime} \\
\end{array}$ & $\begin{array}{c}\text { Horqin } \\
\text { Sandland } \\
\end{array}$ \\
\hline 13 & Woodland & Fixed & $\begin{array}{l}\text { Pinus } \\
\text { tabuliformis }\end{array}$ & 25 & $\begin{array}{c}\text { Aeolian } \\
\text { soil }\end{array}$ & 1396 & $\begin{array}{l}\text { N39 } 18.64^{\prime}, \\
\text { E109 } \\
\end{array}$ & $\begin{array}{c}\mathrm{Mu} \text { Us } \\
\text { Sandland }\end{array}$ \\
\hline 14 & Shrubland & \begin{tabular}{|c|} 
Semi- \\
mobile
\end{tabular} & \begin{tabular}{|l} 
Salix \\
psammophila
\end{tabular} & 39 & $\begin{array}{c}\begin{array}{c}\text { Aeolian } \\
\text { soil }\end{array} \\
\end{array}$ & 1340 & $\begin{array}{l}\text { N39 } 11.13^{\prime}, \\
\text { E109'46.97' } \\
\end{array}$ & \begin{tabular}{|c|} 
Mu Us \\
Sandland
\end{tabular} \\
\hline 15 & Shrubland & $\begin{array}{c}\text { Semi- } \\
\text { mobile }\end{array}$ & H. fruticosum & 27 & $\begin{array}{c}\text { Aeolian } \\
\text { soil }\end{array}$ & 1323 & $\begin{array}{l}\text { N339 } 08.17^{\prime}, \\
\text { E109 } 44.02^{\prime}\end{array}$ & \begin{tabular}{|c|} 
Mu Us \\
Sandland
\end{tabular} \\
\hline 16 & Shrubland & $\begin{array}{c}\text { Semi- } \\
\text { mobile }\end{array}$ & \begin{tabular}{|l} 
Artemisia \\
sieversiana, \\
H. fruticosum
\end{tabular} & 34 & $\begin{array}{l}\text { Aeolian } \\
\text { soil }\end{array}$ & 1336 & $\begin{array}{l}\text { N39 } 08.09^{\prime}, \\
\text { E109 } 32.04^{\prime}\end{array}$ & $\begin{array}{c}\text { Mu Us } \\
\text { Sandland }\end{array}$ \\
\hline 17 & Shrubland & Fixed & Sabina vulgaris & - & $\begin{array}{c}\text { Aeolian } \\
\text { soil }\end{array}$ & 1304 & $\begin{array}{l}\mathrm{N} 38^{\circ} 58.86^{\prime}, \\
\text { E109 } 18.16^{\prime}\end{array}$ & \begin{tabular}{|c|} 
Mu Us \\
Sandland
\end{tabular} \\
\hline 18 & Shrubland & $\begin{array}{c}\text { Semi- } \\
\text { mobile }\end{array}$ & H. fruticosum & 10 & $\begin{array}{c}\text { Aeolian } \\
\text { soil }\end{array}$ & 1386 & $\begin{array}{l}\text { N38 } 8^{\circ} 54.86^{\prime}, \\
\text { E108 } 23.71^{\prime}\end{array}$ & \begin{tabular}{|c|} 
Mu Us \\
Sandland
\end{tabular} \\
\hline
\end{tabular}


Table 1 (continuation)

\begin{tabular}{|c|c|c|c|c|c|c|c|c|}
\hline Plot & Land use & $\begin{array}{l}\text { Sand } \\
\text { dune }\end{array}$ & Vegetation type & \begin{tabular}{|c|} 
Planta- \\
tion \\
duration \\
[years]
\end{tabular} & Soil type & $\begin{array}{c}\text { Altitude } \\
{[\mathrm{m}]}\end{array}$ & Location & Region \\
\hline 19 & Shrubland & $\begin{array}{c}\text { Semi- } \\
\text { mobile }\end{array}$ & Salix cheilophila & - & $\begin{array}{c}\text { Aeolian } \\
\text { soil }\end{array}$ & 1419 & $\begin{array}{l}\text { N38 } 56.67^{\circ}, \\
\text { E108 } 08.75^{\prime}\end{array}$ & \begin{tabular}{|c|} 
Mu Us \\
Sandland
\end{tabular} \\
\hline 20 & Shrubland & Fixed & \begin{tabular}{|l|} 
Caragana \\
korshinskii
\end{tabular} & 12 & $\begin{array}{c}\text { Aeolian } \\
\text { soil }\end{array}$ & 1476 & $\begin{array}{l}\text { N39 } 33.79^{\prime} \\
\text { E108 } \\
\end{array}$ & $\begin{array}{c}\mathrm{Mu} \text { Us } \\
\text { Sandland }\end{array}$ \\
\hline 21 & Grassland & Fixed & \begin{tabular}{|l|}
$\begin{array}{l}\text { Phragmites } \\
\text { communis }\end{array}$ \\
\end{tabular} & - & $\begin{array}{c}\text { Aeolian } \\
\text { soil }\end{array}$ & 1522 & $\begin{array}{l}\mathrm{N} 37^{\circ} 42.40^{\prime}, \\
\mathrm{E} 107^{\circ} 14.18^{\prime}\end{array}$ & $\begin{array}{c}\text { Mu Us } \\
\text { Sandland }\end{array}$ \\
\hline 22 & - & Mobile & $\begin{array}{l}\text { Agriophyllum } \\
\text { squarrosum }\end{array}$ & - & $\begin{array}{c}\text { Aeolian } \\
\text { soil }\end{array}$ & 1545 & $\begin{array}{l}\mathrm{N} 37^{\circ} 42.00^{\prime}, \\
\mathrm{E} 107^{\circ} 13.19^{\prime}\end{array}$ & \begin{tabular}{|c|} 
Mu Us \\
Sandland
\end{tabular} \\
\hline 23 & Woodland & \begin{tabular}{|c|} 
Semi- \\
mobile
\end{tabular} & P. sylvestnis & 12 & $\begin{array}{c}\text { Aeolian } \\
\text { soil }\end{array}$ & 1520 & $\begin{array}{l}\text { N37 } 37^{\circ} 42.49^{\prime}, \\
\text { E107 } 13.85^{\prime}\end{array}$ & \begin{tabular}{|c|} 
Mu Us \\
Sandland
\end{tabular} \\
\hline 24 & Shrubland & \begin{tabular}{|c|}
$\begin{array}{c}\text { Semi- } \\
\text { mobile }\end{array}$ \\
\end{tabular} & Salix mongolica & 12 & $\begin{array}{c}\text { Aeolian } \\
\text { soil }\end{array}$ & 1519 & $\begin{array}{l}\mathrm{N} 37^{\circ} 42.48^{\prime}, \\
\mathrm{E} 107^{\circ} 13.68^{\prime}\end{array}$ & $\begin{array}{c}\text { Mu Us } \\
\text { Sandland }\end{array}$ \\
\hline 25 & Shrubland & \begin{tabular}{|c|}
$\begin{array}{c}\text { Semi- } \\
\text { mobile }\end{array}$ \\
\end{tabular} & & - & & 1501 & & \\
\hline 26 & Shrubland & Fixed & C. korshinskii & 27 & \begin{tabular}{|c|}
$\begin{array}{c}\text { Aeolian } \\
\text { soil }\end{array}$ \\
\end{tabular} & 1478 & $\begin{array}{l}\mathrm{N} 37^{\circ} 43.87^{\prime}, \\
\mathrm{E} 107^{\circ} 13.12^{\prime}\end{array}$ & \begin{tabular}{|c|} 
Mu Us \\
Sandland
\end{tabular} \\
\hline 27 & Shrubland & Fixed & C. $k$ & 11 & $\begin{array}{c}\text { Aeolian } \\
\text { soil }\end{array}$ & 1463 & $\begin{array}{l}\mathrm{N} 37^{\circ} 43.65^{\prime}, \\
\mathrm{E} 107^{\circ} 14.17^{\prime}\end{array}$ & \begin{tabular}{|c|} 
Mu Us \\
Sandland
\end{tabular} \\
\hline 28 & Shrubland & Fixed & C. korshinskii & 7 & $\begin{array}{c}\text { Aeolian } \\
\text { soil }\end{array}$ & 1487 & $\begin{array}{l}\mathrm{N} 37^{\circ} 43.23^{\prime} \\
\mathrm{E} 107^{\circ} 13.89^{\prime}\end{array}$ & $\begin{array}{c}\text { Mu Us } \\
\text { Sandland }\end{array}$ \\
\hline 29 & Shrubland & \begin{tabular}{|c|} 
Semi- \\
mobile
\end{tabular} & $\begin{array}{l}\text { H. fruticosum, } \\
\text { A. sieversiana }\end{array}$ & 14 & $\begin{array}{c}\text { Aeolian } \\
\text { soil }\end{array}$ & 1213 & $\begin{array}{l}\mathrm{N} 40^{\circ} 16.37^{\prime} \\
\mathrm{E} 108^{\circ} 30.64^{\prime}\end{array}$ & $\begin{array}{c}\text { Kubuqi } \\
\text { Desert }\end{array}$ \\
\hline 30 & - & Mobile & - & - & \begin{tabular}{|c|}
$\begin{array}{c}\text { Aeolian } \\
\text { soil }\end{array}$ \\
\end{tabular} & 1075 & $\begin{array}{l}\mathrm{N} 40^{\circ} \\
\mathrm{E} 108\end{array}$ & $\begin{array}{c}\text { Kubuqi } \\
\text { Desert }\end{array}$ \\
\hline 31 & Shrubland & $\begin{array}{c}\text { Semi- } \\
\text { mobile }\end{array}$ & $\begin{array}{l}\text { Arte } \\
\text { ordo }\end{array}$ & 12 & $\begin{array}{c}\text { Aeolian } \\
\text { soil }\end{array}$ & 1349 & $\begin{array}{l}28^{\prime} \\
0.15^{\prime}\end{array}$ & $\begin{array}{c}\text { Tengger } \\
\text { Desert }\end{array}$ \\
\hline 32 & Shrubland & \begin{tabular}{|c|}
$\begin{array}{c}\text { Semi- } \\
\text { mobile }\end{array}$ \\
\end{tabular} & $\begin{array}{l}\text { Hed } \\
\text { scop } \\
\end{array}$ & 12 & $\begin{array}{c}\text { Aeolian } \\
\text { soil }\end{array}$ & 1337 & $\begin{array}{l}\text { N3 } \\
\text { E10 }\end{array}$ & $\begin{array}{c}\text { Tengger } \\
\text { Desert }\end{array}$ \\
\hline 33 & Shrubland & \begin{tabular}{|c|}
$\begin{array}{c}\text { Semi- } \\
\text { mobile }\end{array}$ \\
\end{tabular} & \begin{tabular}{|l|} 
Haloxylon \\
ammodendron
\end{tabular} & 24 & & 1337 & $\begin{array}{l}\mathrm{N} 39^{\circ} 21.09^{\prime} \\
\mathrm{E} 100^{\circ} 07.90^{\prime}\end{array}$ & $\begin{array}{c}\text { Hexi } \\
\text { Desert }\end{array}$ \\
\hline 34 & Shrubland & $\begin{array}{c}\text { Semi- } \\
\text { mobile }\end{array}$ & \begin{tabular}{|l|}
$\begin{array}{l}\text { Calligonum } \\
\text { mongolicunl }\end{array}$ \\
\end{tabular} & 22 & \begin{tabular}{|c|}
$\begin{array}{c}\text { Aeolian } \\
\text { soil }\end{array}$ \\
\end{tabular} & 1356 & $\begin{array}{l}\mathrm{N} 39^{\circ} 21.13^{\prime}, \\
\mathrm{E} 100^{\circ} 08.17^{\prime}\end{array}$ & $\begin{array}{c}\text { Hexi } \\
\text { Desert }\end{array}$ \\
\hline 35 & - & Mobile & - & - & $\begin{array}{c}\text { Aeolian } \\
\text { soil }\end{array}$ & 1384 & $\begin{array}{l}\mathrm{N} 39^{\circ} 21.24^{\prime}, \\
\mathrm{E} 100^{\circ} 07.80^{\prime}\end{array}$ & $\begin{array}{c}\text { Hexi } \\
\text { Desert }\end{array}$ \\
\hline 36 & Shrubland & \begin{tabular}{|c|} 
Semi- \\
mobile
\end{tabular} & \begin{tabular}{|l|} 
Calligonum \\
mongolicunl
\end{tabular} & 9 & \begin{tabular}{|c|}
$\begin{array}{c}\text { Aeolian } \\
\text { soil }\end{array}$ \\
\end{tabular} & ${ }^{14}$ & $\begin{array}{l}\text { N44 } 4^{\circ} 6.31^{\prime}, \\
\text { E088 } \\
\end{array}$ & $\begin{array}{l}\text { Gurbant } \\
\text { nggut Ds }\end{array}$ \\
\hline 37 & Shrubland & \begin{tabular}{|c|} 
Semi- \\
mobile
\end{tabular} & H. ammodendron & 30 & \begin{tabular}{|c|}
$\begin{array}{c}\text { Aeolian } \\
\text { soil }\end{array}$ \\
\end{tabular} & 475 & $\begin{array}{l}\mathrm{N} 44^{\circ} 22.48^{\prime}, \\
\mathrm{E}^{\prime} 87^{\circ} 55.39^{\prime}\end{array}$ & $\begin{array}{l}\text { Gurbant } \\
\text { nggut D }\end{array}$ \\
\hline 38 & Shrubland & $\begin{array}{c}\text { Semi- } \\
\text { mobile }\end{array}$ & H. ammodendron & 18 & $\begin{array}{c}\text { Aeolian } \\
\text { soil }\end{array}$ & 653 & $\begin{array}{l}\mathrm{N}^{\prime} 44^{\circ} 11.73^{\prime}, \\
\mathrm{E} 089^{\circ} 32.75^{\prime}\end{array}$ & $\begin{array}{l}\text { Gurbantu } \\
\text { nggut Ds. }\end{array}$ \\
\hline 39 & $\mathrm{Sh}$ & $\begin{array}{c}\text { Semi- } \\
\text { mobile }\end{array}$ & H. ammodendron & 13 & $\begin{array}{c}\text { Aeolian } \\
\text { soil }\end{array}$ & 678 & $\begin{array}{l}\mathrm{N}^{\prime} 44^{\circ} 56.90^{\prime}, \\
\mathrm{E}^{\circ} 88^{\circ} 32.63^{\prime}\end{array}$ & $\begin{array}{l}\text { Gurbant } \\
\text { nggut D }\end{array}$ \\
\hline
\end{tabular}


ured 5 times and the arithmetic mean value was calculated and recorded. The analysis results of soil PSD were output with regards to the U.S. Soil Taxonomy of soil particle classification: 0-2, 2-50, 50-100, 100-250, 250-500, 5001000 , and $1000-2000 \mu \mathrm{m}$. Further, clay, silt, and sand fractions were defined as $0-2,2-50$, and 50-2000 $\mu \mathrm{m}$, respectively. Additionally, in this study, soil PSD refers to volume-base data.

\subsection{Data processing}

Based on the soil PSD data, volume-based fractal dimensions of soil PSD were subsequently calculated according to the fractal model of Wang et al. (2005) and Gui et al. (2010). The equation is expressed as:

$$
\frac{V\left(r<R_{i}\right)}{V_{T}}=\left(\frac{R_{i}}{R_{\max }}\right)^{3-D},
$$

where $r$ is the soil particle size, $R_{i}$ is the soil particle size of grade $i, R_{\max }$ is the maximum value of soil particle size, $V\left(r<R_{i}\right)$ is the volume of soil particle size less than $R_{i}, V_{T}$ is the total volume of soil particles, $D$ is the volume-based fractal dimension.

In this study, the field plot and sub-plot were used as the basic study unit. The mean values of soil PSD data and fractal dimensions were identified for statistical analysis. Linear regression, hierarchical cluster and correlation analysis were performed to identify the relationships between $D$ and soil particle fractions. Correlation analysis passed a 2-tailed test to distinguish the significant difference when evaluated at the level of $P<0.01$. All statistical analyses were conducted using the SPSS software (Version 17.0).

\section{RESULTS AND DISCUSSIONS}

\subsection{Soil PSD and fractal dimension of soil PSD}

Based on the data measured by the Malvern Laser Diffraction Instrument, 39 groups of soil PSD were classified (Table 2). There were considerable differences in soil PSD among the 39 field plots. Furthermore, although there was large variability due to the differences in climate, desertification degree, as well as vegetation restoration solutions, the dominant soil particle classification was the size of sand particles. Among them, fine sand and medium sand dominate sand particles, accounting for 39.59 and $22.02 \%$ of total fractions, respectively. Accordingly, the content of clay fractions was much lower, ranging from 0.00 to $8.18 \%$ (mean value of $1.63 \%$ ). Soils of this nature are classified as aeolian soil, which is commonly found in arid, semiarid, and dry sub-humid regions of northern China. This finding resulted from long term wind-induced soil erosion and was consistent with the earlier 
Table 2 Soil PSD and fractal dimensions of soil PSD of different field station

\begin{tabular}{|c|r|r|r|c|}
\hline Plot & Clay $[\%]$ & \multicolumn{1}{|c|}{ Silt $[\%]$} & \multicolumn{1}{|c|}{ Sand $[\%]$} & $D$ \\
\hline 1 & $2.86 \pm 0.25$ & $33.91 \pm 7.99$ & $59.88 \pm 8.24$ & $2.4182 \pm 0.0701$ \\
2 & $3.33 \pm 0.36$ & $38.45 \pm 0.84$ & $58.22 \pm 0.71$ & $2.3606 \pm 0.0314$ \\
3 & $3.11 \pm 0.54$ & $37.83 \pm 5.10$ & $59.05 \pm 5.33$ & $2.3880 \pm 0.1572$ \\
4 & $1.34 \pm 0.11$ & $15.49 \pm 2.45$ & $83.16 \pm 2.40$ & $2.3376 \pm 0.0057$ \\
5 & $1.86 \pm 0.62$ & $14.65 \pm 6.62$ & $83.49 \pm 7.19$ & $2.4007 \pm 0.0794$ \\
6 & $1.51 \pm 0.57$ & $15.55 \pm 5.86$ & $82.95 \pm 6.36$ & $2.3658 \pm 0.0105$ \\
7 & $1.13 \pm 0.19$ & $10.66 \pm 1.50$ & $88.21 \pm 1.69$ & $2.3968 \pm 0.0252$ \\
8 & $1.50 \pm 0.50$ & $15.67 \pm 5.23$ & $82.84 \pm 5.73$ & $2.4414 \pm 0.0464$ \\
9 & $2.90 \pm 1.25$ & $24.96 \pm 8.95$ & $72.14 \pm 10.21$ & $2.5153 \pm 0.0468$ \\
10 & $0.79 \pm 0.15$ & $11.32 \pm 1.47$ & $87.89 \pm 1.57$ & $2.3695 \pm 0.0214$ \\
11 & $0.75 \pm 0.16$ & $7.76 \pm 0.86$ & $91.48 \pm 1.01$ & $2.2655 \pm 0.0298$ \\
12 & $0.08 \pm 0.14$ & $0.67 \pm 1.15$ & $99.25 \pm 1.29$ & $2.1685 \pm 0.0855$ \\
13 & $3.17 \pm 0.12$ & $27.65 \pm 2.31$ & $69.18 \pm 2.23$ & $2.5123 \pm 0.0025$ \\
14 & $1.61 \pm 0.23$ & $16.24 \pm 2.22$ & $82.15 \pm 2.40$ & $2.4062 \pm 0.0190$ \\
15 & $1.06 \pm 0.27$ & $9.50 \pm 1.51$ & $89.44 \pm 1.74$ & $2.3365 \pm 0.0386$ \\
16 & $1.54 \pm 0.27$ & $16.43 \pm 2.57$ & $82.02 \pm 2.81$ & $2.3991 \pm 0.0281$ \\
17 & $3.15 \pm 0.41$ & $38.67 \pm 4.31$ & $58.18 \pm 4.29$ & $2.5253 \pm 0.0172$ \\
18 & $0.71 \pm 0.09$ & $6.09 \pm 0.66$ & $93.20 \pm 0.73$ & $2.2580 \pm 0.0182$ \\
19 & $2.24 \pm 0.40$ & $18.97 \pm 2.21$ & $78.90 \pm 2.51$ & $2.4717 \pm 0.0128$ \\
20 & $2.12 \pm 0.40$ & $18.97 \pm 2.21$ & $78.90 \pm 2.51$ & $2.4480 \pm 0.0274$ \\
21 & $0.77 \pm 0.35$ & $6.48 \pm 0.10$ & $92.75 \pm 0.26$ & $2.2514 \pm 0.0745$ \\
22 & $0.09 \pm 0.16$ & $1.46 \pm 2.48$ & $98.44 \pm 2.64$ & $2.0810 \pm 0.0421$ \\
23 & $0.32 \pm 0.37$ & $4.54 \pm 4.34$ & $95.14 \pm 4.70$ & $2.1742 \pm 0.0848$ \\
24 & $0.24 \pm 0.04$ & $5.92 \pm 0.05$ & $93.84 \pm 0.09$ & $2.1901 \pm 0.0684$ \\
25 & $0.49 \pm 0.35$ & $5.76 \pm 0.65$ & $93.76 \pm 0.92$ & $2.2641 \pm 0.0283$ \\
26 & $6.58 \pm 1.49$ & $62.41 \pm 10.87$ & $31.01 \pm 12.35$ & $2.6351 \pm 0.0363$ \\
27 & $4.08 \pm 0.03$ & $30.94 \pm 2.92$ & $64.99 \pm 2.89$ & $2.5484 \pm 0.0027$ \\
28 & $3.34 \pm 0.19$ & $50.06 \pm 4.17$ & $46.60 \pm 4.36$ & $2.5408 \pm 0.0109$ \\
29 & $2.68 \pm 0.06$ & $9.81 \pm 0.70$ & $87.51 \pm 0.75$ & $2.4503 \pm 0.0010$ \\
30 & $0.00 \pm 0.00$ & $0.00 \pm 0.00$ & $100.00 \pm 0.00$ & $2.1766 \pm 0.0217$ \\
31 & $0.17 \pm 0.30$ & $4.21 \pm 3.74$ & $95.62 \pm 3.95$ & $2.2407 \pm 0.0402$ \\
32 & $0.82 \pm 0.12$ & $5.48 \pm 0.71$ & $93.70 \pm 0.76$ & $2.2672 \pm 0.0221$ \\
33 & $2.81 \pm 0.55$ & $10.37 \pm 0.94$ & $86.82 \pm 1.49$ & $2.4535 \pm 0.0297$ \\
34 & $1.83 \pm 0.09$ & $5.96 \pm 0.49$ & $92.21 \pm 0.57$ & $2.3801 \pm 0.0090$ \\
35 & $0.11 \pm 0.18$ & $0.22 \pm 0.38$ & $99.67 \pm 0.57$ & $2.1678 \pm 0.0804$ \\
36 & $0.61 \pm 0.55$ & $3.99 \pm 2.87$ & $95.39 \pm 3.42$ & $2.2558 \pm 0.0551$ \\
37 & $1.56 \pm 0.04$ & $10.84 \pm 2.54$ & $87.61 \pm 2.54$ & $2.3790 \pm 0.0087$ \\
39 & $0.30 \pm 0.41$ & $2.99 \pm 2.63$ & $96.72 \pm 2.95$ & $2.1525 \pm 0.1334$ \\
& $0.00 \pm 0.00$ & $0.18 \pm 0.26$ & $99.82 \pm 0.26$ & $2.2059 \pm 0.0607$ \\
\hline & & & & \\
\hline
\end{tabular}


studies on the basic mechanical composition of aeolian soil in China ( $\mathrm{Su}$ et al. 2004, Ding 2010).

Table 2 shows the calculated $D$ values when Eq. 1 was applied to each soil data set. The $D$ values covered a wide range from 2.0810 to 2.6351. Although changes in $D$ values varied significantly, there was a clear tendency among the different field sampling plots that $D$ values were significantly affected by the degree of desertification in the investigated area. Generally, wind-induced desertification results in selective removal characterized by clay and silt particle loss; during this coarsening process, $D$ values kept decreasing with the increasing degree of soil desertification. Accordingly, in this study, the fixed sand dunes usually had relatively higher $D$ values (mean value of 2.4676), whereas lower $D$ values were always found in the plots of mobile sand dunes (mean value of 2.1485). Furthermore, semi-mobile sand dunes had medium $D$ values (mean value of 2.3243) between the fixed and mobile sand dunes.

Vegetation solutions, not only natural but also artificial, had considerable effects on soil PSD and $D$ values as well. Overall, anti-desertification solutions had considerable increases in the fine fractions, and soil PSD recovered more as vegetation ages increased (e.g., Plots 26-28). These functions in soil PSD improvement and $D$ value increases are addressed by two activities. On one hand, vegetation restoration solutions consumed erosive forces and carriage ability by increasing aerodynamic roughness lengths and threshold wind velocity, allowing the fine particles to be preserved. Furthermore, biological and physiological activity by vegetation such as photosynthesis, azotification, and litters decomposition also help soil systems to prosper.

In short, fractal dimensions of soil PSD can provide additional information regarding soil degradation among different stages of desertification as well as different solutions for anti-desertification efforts.

\subsection{Relationships between fractal dimensions and soil particle contents}

Through a log-log transformation, simple linear regression and correlation analyses were performed to identify the strength of correlations between $D$ values and the contents of clay, silt, and sand particles (Fig. 1, Table 3). The determination coefficients $\left(R^{2}\right)$ of the linear regressions were high and ranged from 0.50 to 0.81 ; all the regression analyses passed a two-tailed test indicating the significant difference when evaluated at $p<0.01$. Specifically, a significant positive correlation occurred between logarithmic $D$ values and clay fractions with $R^{2}=0.81, P<0.01$ (Fig. 1A). Figure 1B showed a significant positive correlation between logarithmic $D$ values and silt fractions $\left(R^{2}=0.59, P<0.01\right)$. By contrast, a significant negative correlations was observed, with $R^{2}=0.50, P<0.01$ between logarithmic $D$ values and sand 

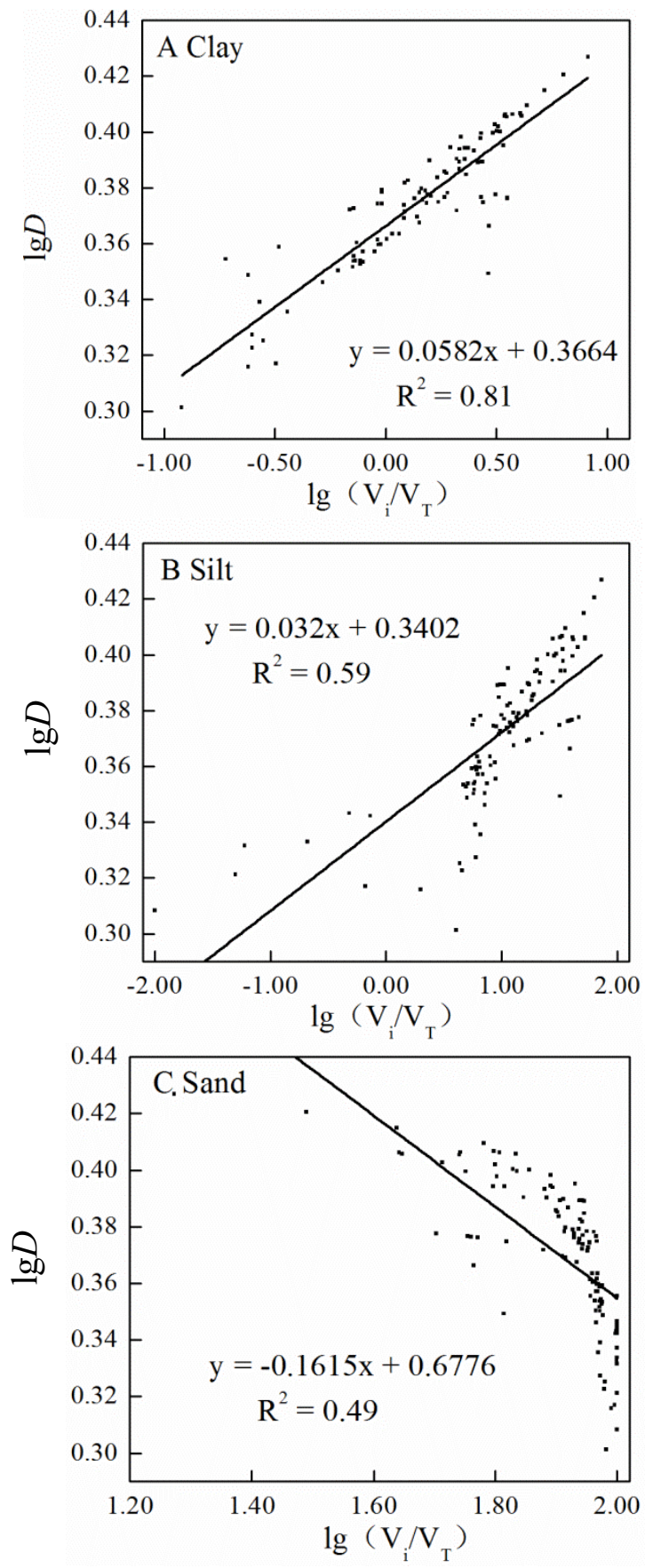

Fig. 1. Relationships between $D$ values and soil PSD. 
Table 3

Correlation coefficients between $D$ values and soil PSD through a logarithmic transformation

\begin{tabular}{|c|c|c|c|}
\hline Soil properties & Clay & Silt & Sand \\
\hline Correlation coefficients & $0.900 *$ & $0.769 *$ & $-0.704 *$ \\
\hline
\end{tabular}

*) correlation is significant at the 0.01 level (2-tailed)

Table 4

Plots division through a hierarchical cluster analysis

\begin{tabular}{|c|c|}
\hline Type & \multicolumn{1}{|c|}{ Plots } \\
\hline I & $1,3,9,13,17,26-28$, \\
II & $2,4-8,10,14,16,19-20,29,33-34,37$ \\
III & $11-12,15,18,21-25,30-32,35-36,38-39$ \\
\hline
\end{tabular}

fractions (Fig. 1C). This finding indicated that the selected removal of clay and silt fractions resulted in decreasing $D$ values. For instance, the highest $D$ value was found in Plot 26 which had the highest clay and silt contents (6.58 and $62.41 \%$ ) and lowest sand content (31.01\%); the lowest $D$ value corresponded to Plot 22, which had lower clay and silt contents (0.09 and 1.46\%) and higher sand content (98.44\%). To further identify the relationship between $D$ values and desertification, we conducted a hierarchical cluster analysis. The study plots were divided into three types based on $D$ values (Table 4). Combining Table 1, the result indicated that Types I and III mainly covered fixed and mobile sand dunes, respectively. Type II was a transitional type, which was composed primarily of semi-mobile dunes. This tendency was also basically consistent with $D$ value distribution among area with different degrees of desertification.

Studies regarding soil fractal features are numerous and have led to a much greater understanding of soil environment changes affected by wind erosion in desertified regions. However, although these studies are helpful for estimating variations in soil PSD as well as other soil properties, such as soil organic carbon, nutrients, moisture characteristics, and hydraulic conductivities, fractal models of soil PSD have not been explored in full. Spatial scale effects are one of the most important limitations. In former studies, field investigation always covered an independent and limited area, such as a plateau area, specific mountain, or and even an administrative region (Wang et al. 2006, Fu et al. 2009, Gao et al. 2014a, b). Whereas heterogeneity is a ubiquitous feature of ecological systems; soil properties including soil PSD usually change spatially and temporally (Millán 2007). Therefore, accurate 
estimations of soil PSD information with fractal models when scaling up from plots to regions, while maintaining meaningful field-scale process details, remains a challenge. In this study, the sampling plots were all arid, semi-arid, and dry sub-humid regions in northern China. From the east to west, the straight-line distance is more than $3000 \mathrm{~km}$. In this large spatial scale, fractal dimensions still significantly correlated with soil particles, and is demonstrated to be sensitive to soil texture and wind-induced desertification. Breaking the restriction of fractal behavior, it is possible to make reliable assessment of variations in soil PSD indicating soil environment changes at different spatial scales and especially on a large spatial scale.

\section{CONCLUSION}

In desertified regions of northern China, wind-induced land desertification and vegetation restoration significantly changed the soil PSD. The predominant soil particle sizes concentrated in fine and medium sand fraction classifications accounted for more than $50 \%$ of total volume. Fractal analysis showed $D$ values covered a wide range, from 2.0810 to 2.6351. Based on linear regression and correlation analysis, significant positive correlations occurred between $\log$ arithmic $D$ values and clay and silt particle contents $\left(R^{2}=0.81\right.$ and $\left.0.59, P<0.01\right)$, in addition to significant negative correlations between logarithmic $D$ values and sand particle content $\left(R^{2}=0.50\right.$, $P<0.01)$. Hierarchical cluster analysis showed that the sorted plots were similar to sand dune types indicating desertification degree. On a large spatial scale, $D$ was still sensitive to the desertification processes by characterizing soil PSD and its variations. It was suggested that $D$ could be used as a reliable and quantitative parameter to monitor soil systems, and significantly implied land desertification. This improved information contributes to a better understanding of desertification processes in China.

Acknowledgements. We gratefully acknowledged our colleagues in Yanchi Research Station for their generous help in field-based investigation and Jon Gartner (New York, U.S.A.) for the language editing on the earlier edition of this manuscript as well as the great cooperation with the Environmental Protection and Forestry Bureau of Yanchi County, Ningxia Hui Autonomous Region. This work was financial supported by the Fundamental Research Funds for the Central Universities (BLX2014-15 and BJFUTD 2011-04), the National Basic Research Program of China (2013CB429906), and the National Technology and Science Support Program of P.R. China (2012BAD16B02). 


\section{References}

Arrouays, D., N. Saby, C. Walter, B. Lemercier, and C. Schvartz (2006), Relationships between particle-size distribution and organic carbon in French arable topsoils, Soil Use Manage. 22, 1, 48-51, DOI: 10.1111/j.1475-2743.2006. 00020.x.

Buchan, G.D. (1989), Applicability of the simple lognormal model to particle-size distribution in soils, Soil Sci. 147, 3, 155-161, DOI: 10.1097/00010694198903000-00001.

Chen, X.H., and Z.H. Duan (2009), Changes in soil physical and chemical properties during reversal of desertification in Yanchi County of Ningxia Hui autonomous region, China, Environ. Geol. 57, 5, 975-985, DOI: 10.1007/ s00254-008-1382-1.

Chen, Y., and H. Tang (2005), Desertification in north China: Background, anthropogenic impacts and failures in combating it, Land Degrad. Develop. 16, 4, 367-376, DOI: 10.1002/1dr.667.

Crawford, J.W., B.D. Sleeman, and I.M. Young (1993), On the relation between number-size distributions and the fractal dimension of aggregates, Eur. J. Soil Sci. 44, 4, 555-565, DOI: 10.1111/j.1365-2389.1993.tb02321.x.

Ding, G.D. (2010), Blown Sand Physics, China Forestry Publishing, Beijng (in Chinese).

Duan, Z.H., H.L. Xiao, X.R. Li, Z.B. Dong, and G. Wang (2004), Evolution of soil properties on stabilized sands in the Tengger Desert, China, Geomorphology 59, 1-4, 237-246, DOI: 10.1016/j.geomorph.2003.07.019.

Fu, H., S.F. Pei, C.G. Wan, and R.E. Sosebee (2009), Fractal dimension of soil particle size distribution along an altitudinal gradient in the Alxa rangeland, western Inner Mongolia, Arid Land Res. Manage. 23, 2, 137-151, DOI: 10.1080/15324980902813658.

Gao, G.L., G.D. Ding, B. Wu, Y.Q. Zhang, S.G. Qin, Y.Y. Zhao, Y.F., Bao, Y.D. Liu, L. Wan, and J.F. Deng (2014a), Fractal scaling of particle size distribution and relationships with topsoil properties affected by biological soil crusts, PLoS One 9, 2, e88559, DOI: 10.1371/journal.pone.0088559.

Gao, G.L., G.D. Ding, Y.Y. Zhao, B. Wu, Y.Q. Zhang, S.G. Qin, Y.F. Bao, M.H. Yu, and Y.D. Liu (2014b), Fractal approach to estimating changes in soil properties following the establishment of Caragana korshinskii shelterbelts in Ningxia, NW China, Ecol. Indic. 43, 236-243, DOI: 10.1016/ j.ecolind.2014.03.001.

Gui, D.W., J.Q. Lei, F.J. Zeng, G.J. Mu, J.T. Zhu, H. Wang, and Q. Zhang (2010), Characterizing variations in soil particle size distribution in oasis farmlands - A case study of the Cele Oasis, Math. Comput. Model. 51, 11-12, 13061311, DOI: 10.1016/j.mcm.2009.10.035.

Hillel, D. (1980), Fundamentals of Soil Physics, Academic Press, New York, $413 \mathrm{pp}$. 
Hwang, S.I., K.P. Lee, D.S. Lee, and S.E. Powers (2002), Models for estimating soil particle-size distributions, Soil Sci. Soc. Am. J. 66, 4, 1143-1150, DOI: 10.2136/sssaj2002.1143.

Jiao, F., Z.M. Wen, and S.S. An (2011), Changes in soil properties across a chronosequence of vegetation restoration on the Loess Plateau of China, Catena 86, 2, 110-116, DOI: 10.1016/j.catena.2011.03.001.

Jin, Z., Y.S. Dong, Y.C. Qi, W.G. Liu, and Z.S. An (2013), Characterizing variations in soil particle-size distribution along a grass-desert shrub transition in the Ordos Plateau of Inner Mongolia, China, Land Degrad. Develop. 24, 2, 141-146, DOI: 10.1002/1dr.1112.

Li, X.R., M.Z. He, Z.H. Duan, H.L. Xiao, and X.H. Jia (2007), Recovery of topsoil physicochemical properties in revegetated sites in the sand-burial ecosystems of the Tengger Desert, northern China, Geomorphology 88, 3-4, 254265, DOI: 10.1016/j.geomorph.2006.11.009.

Liu, X., G.C. Zhang, G.C. Heathman, Y.Q. Wang, and C.H. Huang (2009), Fractal features of soil particle-size distribution as affected by plant communities in the forested region of Mountain Yimeng, China, Geoderma 154, 1-2, 123130, DOI: 10.1016/j.geoderma.2009.10.005.

Lobe, I., W. Amelung, and C.C. Du Preez (2001), Losses of carbon and nitrogen with prolonged arable cropping from sandy soils of the South African Highveld, Eur. J. Soil Sci. 52, 1, 93-101, DOI: 10.1046/j.1365-2389.2001. t01-1-00362.x.

Mandelbrot, B.B. (1967), How long is the coast of Britain? Statistical self-similarity and fractional dimension, Science 156, 3775, 636-638, DOI: 10.1126/ science.156.3775.636.

Mandelbrot, B.B. (1983), The Fractal Geometry of Nature, Freeman, New York, $468 \mathrm{pp}$.

Millán, H. (2007), Scale cutoffs and the limits of fractal soil structure, Int. Agrophys. 21, 2, 169-172.

Nemes, A., J.H.M. Wösten, A. Lilly, and J.H.O. Voshaar (1999), Evaluation of different procedures to interpolate particle-size distributions to achieve compatibility within soil databases, Geoderma 90, 3-4, 187-202, DOI: 10.1016/S0016-7061(99)00014-2.

Reynolds, R.L., M. Reheis, J. Yount, and P. Lamothe (2006), Composition of aeolian dust in natural traps on isolated surfaces of the central Mojave Desert - Insights to mixing, sources, and nutrient inputs, J. Arid Environ. 66, 1, 42-61, DOI: 10.1016/j.jaridenv.2005.06.031.

SFA (2011), A Bulletin of Status Quo of Desertification and Sandification in China, State Forestry Administration of the People's Republic of China, Bejing, http://www.greentimes.com/green/econo/hzgg/ggqs/content/2011-01/05/ content_114232.htm (in Chinese). 
Skaggs, T.H., L.M. Arya, P.J. Shouse, and B.P. Mohanty (2001), Estimating particle-size distribution from limited soil texture data, Soil Sci. Soc. Am. J. 65, 4, 1038-1044, DOI: 10.2136/sssaj2001.6541038x.

Su, Y.Z., H.L. Zhao, W.Z. Zhao, and T.H. Zhang (2004), Fractal features of soil particle size distribution and the implication for indicating desertification, $\mathrm{Ge}$ oderma 122, 1, 43-49, DOI: 10.1016/j.geoderma.2003.12.003.

Turcotte, D.L. (1986), Fractals and fragmentation, J. Geophys. Res. 91, B2, 19211926, DOI: 10.1029/JB091iB02p01921.

Tyler, S.W., and S.W. Wheatcraft (1990), Fractal processes in soil water retention, Water Resour. Res. 26, 5, 1047-1054, DOI: 10.1029/WR026i005p01047.

Tyler, S.W., and S.W. Wheatcraft (1992), Fractal scaling of soil particle-size distribution: analysis and limitations, Soil Sci. Soc. Am. J. 56, 2, 362-369, DOI: 10.2136/sssaj1992.03615995005600020005x.

UNEP (1994), United Nations Convention to combat desertification in those countries experiencing serious drought and/or desertification, particularly in Africa, United Nations Environment Programme for the Convention to Combat Desertification (CCD), Interim Secretariat for the CCD, Switzerland, Geneva, $71 \mathrm{pp}$.

Wang, G.L., S.L. Zhou, and Q.G. Zhao (2005), Volume fractal dimension of soil particles and its applications to land use, Acta Pedol. Sin. 42, 4, 545-550.

Wang, X.D., M.H. Li, S.Z. Liu, and G.C. Liu (2006), Fractal characteristics of soils under different land-use patterns in the arid and semiarid regions of the Tibetan Plateau, China, Geoderma 134, 1-2, 56-61, DOI: 10.1016/j.geoderma. 2005.08.014.

Wang, X.M., F.H. Chen, E. Hasi, and J.C. Li (2008), Desertification in China: An assessment, Earth-Sci. Rev. 88, 3-4, 188-206, DOI: 10.1016/j.earscirev. 2008.02.001.

Zha, Y., and J. Gao (1997), Characteristics of desertification and its rehabilitation in China, J. Arid Environ. 37, 3, 419-432, DOI: 10.1006/jare.1997.0290.

Received 15 January 2014 Received in revised form 20 September 2014 Accepted 18 November 2014 